\title{
Studies on Reductive Pauson-Khand Reaction Using Cobaltcarbonyls with Water
}

\author{
Hec-Yoon Lee, ${ }^{\star}$ Mihyun An, and Jeong-Hun Sohn \\ Center for Molecular Design and Synthesis, Department of (hemistry \& BK2l School of Molecular Science, \\ Korea Advanced Institute of Science and Technologv, Daejeon 305-701, Korea \\ Received Februar 25, 2003
}

Key Words : Pauson-Khand reaction, Reduction, Water

During the course of our synthetic program toward total synthesis of arteminolide, 'highly substituted cyclopentenone. A emerged as a good starting point of the total synthesis and was readily prepared from $\mathbf{B}$ through Pauson-Khand reaction $(\mathrm{PKR})^{2}$ followed by reductive cleavage of the ether bridge. During a gram scale I'KR, A was produced as a byproduct and even became the major product when hydrated $\mathrm{N}$-oxides instead of anhydrous ones were used in the PKR (Scheme 1).

While reductive $P K R$ has been observed in the past as one of the side reactions during development of modified PKR using various solvents or additives ${ }^{3,4}$ including water, there were only few reports of reductive PKR being the major process during Pauson-Khand reaction. ${ }^{4}{ }^{3}$ Though the presence of proton sources in the reductive PKR reaction appeared to be vital to the reduction process among all the reported examples, ${ }^{8-10}$ these proton sources have not been studied much as a reagent for the reductive $\mathrm{P} K \mathrm{KR}$. Therefore, we became interested in developing a reduction system from $\mathrm{Co}_{2}(\mathrm{CO})_{8}$ and $\mathrm{H}_{2} \mathrm{O}$ with or without being associated with the PKR.

Herein we like to report a reductive PKR using water as a part of the reducing agent with $\mathrm{CO}_{2}(\mathrm{CO})_{8}$. Since our initial study on the $\mathrm{N}$-oxide promoted PKR showed that addition of water other than hydrates of $\mathrm{N}$-oxides did not promote the reductive PKR, we tumed our attention to the classical PKR. After exploring several reaction conditions using water for reductive PKR with substrate 1, we found out that the choice of solvent and the amount of water is critical for the reductive PKR. DME turned out to be the best solvent for the reductive $P K R$ and the effective reduction required more than 20 equivalents of water (Scheme 2). Acetonitrile was not as effective as expected though Pagenkopf reported successful application of wet acetonitrile for reductive PKR. ${ }^{8}$

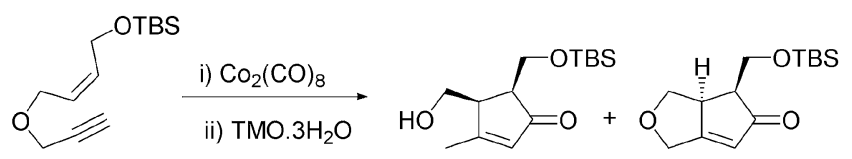

B A

Scheme 1

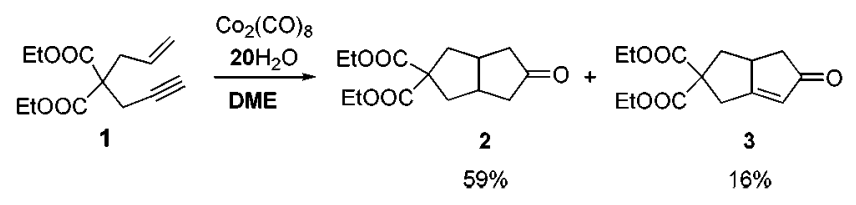

Scheme 2
Apparently, Pagenkopfs reductive PKR followed a quite different route to the other reductive PKRs.

Since $\mathrm{Co}_{2}(\mathrm{CO})_{8}$ was used both for the PKR and reduction, more than one equivalent of $\mathrm{CO}_{2}(\mathrm{CO})_{8}$ would be required to complete J'KR and the reduction of the J'KR product. We hoped that substoichiometric amounts of $\mathrm{CO}_{2}\left(\mathrm{CO}_{8}\right.$ might be sufficient enough to complete the PKR and reduction since it has been already demonstrated that $\mathrm{PKR}$ was accomplished with substoichiometric use of $\mathrm{CO}_{2}\left(\mathrm{CO}_{8}{ }_{8}{ }^{\prime \prime}\right.$ and the cobalt species generated after the reduction could be recycled back to $\mathrm{CO}_{2}(\mathrm{CO})_{8}{ }^{12}$ or directly to the reducing species ${ }^{13}$ if $\mathrm{CoH}(\mathrm{CO})_{4}$ or related compound was involved in the reduction. To confirm our expectation, we examined the reductive $\mathrm{PKR}$ under three different conditions. The first reaction condition used one equivalent of $\mathrm{Co}_{2}(\mathrm{CO})_{8}$ and the second one used 1.5 equivalent of $\mathrm{Co}_{2}(\mathrm{CO})_{8}$ to provide sufficient amount of reagents for both $\mathrm{PKR}$ and the reduction. The last one used 0.5 equivalent of $\mathrm{Co}_{4}(\mathrm{CO})_{12}$ with a hope that it could provide the same amount of reactive agents as one equivalent of $\mathrm{Co}_{2}(\mathrm{CO})_{8}$ in the reaction.

lable 1 summarized the reductive PKR of various substrates under three different conditions. As we anticipated, one equivalent of $\mathrm{Co}_{2}(\mathrm{CO})_{8}$ was sufficient enough to carry out both $\mathrm{PKR}$ and reduction completely (entry 13). Since it required at least 0.3 equivalent of $\mathrm{Co}_{2}(\mathrm{CO})_{8}$ to effectively complete $\mathrm{P}^{\mathrm{KKR}}{ }^{11}$ the reduction was completed with less than 0.7 equivalent of $\mathrm{Co}_{2}(\mathrm{CO})_{8}$ but still required more than 0.5 equivalent of $\mathrm{CO}_{2}(\mathrm{CO})_{8}$ (entry 15). It appeared that nitrogen in the substrate facilitated the regeneration of the reactive species for the reduction. ' For other substrates, when 1.5 equivalent of $\mathrm{Co}_{2}(\mathrm{CO})_{8}$ was used to ensure the existence of sufficient amount of reducing species generated from $\mathrm{Co}_{2}(\mathrm{CO})_{8}$, overall yjeld of the products as well as the ratio of the reduced products to the PKR products was improved (entry $2,5,8,17$ ). In the case of the substrate with an ether linkage, overall yield of the reduced products was improved. However, the amount of reductive ether cleavage product was not affected much and only the amount of the 1,4reduction product increased as more $\mathrm{Co}_{2}\left(\mathrm{CO}_{8}\right.$ was used (entry 16, 17). These results strongly suggest that there might be two distinct reducing species produced in the reaction; one is directly generated from $\mathrm{Co}_{2}(\mathrm{CO})_{8}$ and the other is generated from a cobalt intermediate from PKR. Entry 19 showed that the reduction most likely proceeds after the I'KR as reduction of conjugated olefin proceeded directly from $\mathrm{CO}_{2}(\mathrm{CO})_{8}$ and water without being associated 
Table 1. Reductive PKR under various conditions

Entry Substratc Conditions ${ }^{\prime 2}$

"A $0.5 \mathrm{M}$ solution of the substrate in DMS: was retluxed with cobalt carbonvls and water under $\mathrm{Ar}$ atm. Ior $2 \mathrm{hr}$. condition $\mathrm{A}: \mathrm{Co}(\mathrm{CO})_{8}(\mathrm{I}$ eq $)$.

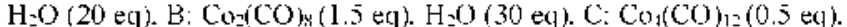
$\mathrm{II}_{2} \mathrm{O}(20 \mathrm{cq}) . \mathrm{D}: \mathrm{CO}_{2}\left(\mathrm{CO}_{\mathrm{s}}(0.75 \mathrm{cq}) . \mathrm{I}_{2} \mathrm{O}(20 \mathrm{cq})\right.$ "isolated sicld. "NII the products were either characterized fully by. 'II-NMR. "C-NMR or were known compounds. ${ }^{\text {"i }}$

with PKR. The reduction was also sensitive to the substitution pattern and the tether variation of substrates. When a substrate with an internal alky ne was used for reductive PKR that produced a tetra-substituted enone. the subsequent reduction became very slow (entry 7-9). Even a substitution at the positions other than olefin affected the reduction (entry 4-6). When the length of the tether became longer. the reduction after PKR became very slow (entry 10-12). This reactivity pattern is similar to the reported reductive PKR and the reaction appears to be more effective than the reported ones as combined overall yields of products of PKR and reductive PKR were high with no other byproduct formation. ${ }^{10}$ In the case of $\mathrm{CO}_{4}(\mathrm{CO})_{1} \_.0 .5$ equivalent of $\mathrm{Co}_{4}(\mathrm{CO})_{12}$ was sufficient enough to complete the reductive PKR and to our surprise. the result was very similar to the result of reductive $\mathrm{PKR}$ using 1.5 equivalent of $\mathrm{CO}_{2}(\mathrm{CO})_{8}$ (entry 3.6. 9. 12, 18) rather than the result from reactions with one equivalent of $\mathrm{Co}_{-}(\mathrm{CO})_{8}$. This is a strong indication that $\mathrm{CO}_{4}(\mathrm{CO})_{1}$. instead of being transformed into $\mathrm{Co}_{-}(\mathrm{CO})_{8}$ prior to the reaction with substrates ${ }^{1+}$ is converted into a dicobalt-alkyne complex with substrates and leaves another cobalt complex that is readily transformed into $\mathrm{Co}_{2}(\mathrm{CO})_{4}$.

In summary, we were able to clarify that the reductive PKR requires not only a proton source but also a polar solvent with good coordinating ability as reductive PKR with water and $\mathrm{CO}_{2}(\mathrm{CO})_{8}$ was found to be most effective in DME and the best result was obtained when 1 equivalent of $\mathrm{Co}_{2}(\mathrm{CO})_{\times}$with 20 equivalent of water were used for the reductive PKR. Only half equivalent use of $\mathrm{Co}_{1}(\mathrm{CO})_{12}$ was found to be as effective for the reductive $\mathrm{PKR}$ as $\mathrm{CO}_{2}(\mathrm{CO})_{*}$. When compared to $\mathrm{Co}_{1}(\mathrm{CO})_{22}$-isopropanol system. ${ }^{(i)}$ the current system using water in DME not only afforded better yield of reductive PKR products with no other products such as reductive cyclization product. but also required less amount of cobalt reagents. Since $\mathrm{CoH}(\mathrm{CO})$. 1 was reported to show a reverse isotope effect ${ }^{12}$ while we and others did not observe that effect. the real reducing species in the reductive PKR seems to be different from $\mathrm{CoH}(\mathrm{CO})_{1}$ and the nature of the reducing species varies depending on the reaction condition. The sensitive nature of the current reducing system could provide an unprecedented chemoselective reduction.

Acknow ledgments. We thank Dr. J. M. Nuss and professor $\mathrm{N}$. Jcong for hclpful discussion.

\section{References}

1. I.ec, S.-II.; Kim. M.-I.; Bok. S. H.: I.ec. H.: Kwon, B.-M.; Shin, J.: Seo. Y.J. Oig (hem. 1998.63.7111.

2. (a) Brummond. K. M.: Kent. I. L. Tetrahedron 2000. 56. 3263. (b) Shambayati. S.: Crowe. W. E.: Schreiber. S. L. Tetrahedron Lett. 1990, 31, 5289. (c) Jcong. N.: Chung. Y. K.: I.ce. B. Y.: I.ce, S. II.: Yoo, S. Smktt 1991, 204.

3. (a) Thommen. M.: Vereteno: A. I.: Guidetti-Girept. R.: Keese. R. Hele Chin ito 1996. 79. 461. (b) Kerr. W. J.: McLaughlin. M.: Pauson. P. L.: Robertson. S. M. Chem. Commun 1999. 2171.

4. (a) Jeong. N.: Yoo. S.: Lee. S. I.: Lee. S. H.: Chung. Y. K. Fumbetron Lett. 1991, 32, 2137. (b) Almansa. C.: Carceller, E.: Garcia. M. J..: Torrents, A.: Serratosa. F. Swh. Common. 1988. I8, 381. (c) Chung. Y. K.: I ce, B. Y.: Jcong. N.: Iudecet, M.: P'atson. P. L. O,ganometallics 1993. 12. 220. (d) Simonian. S. O.: Smit. W. A.: Gybin. A. S.: Shashkor. A. S.: Mikaelian. (i. S.: Tarasox. V. A.: Ibragimox. I. I.: Caple. R.: Froen. D. E. Fetrafuedron Lett. 1986, 27, 1245.

5. Son, S. U.; I.ee, S. I.: Clumg. Y. K.: Kim. S. W.: Hyyn. T. Ory. Lett. 2002. f, 277 .

6. Smit. W. A.: Simonyan. S. O.: Tarasoy. V. A.: Mikaelian. G. S.: Gybin. A. S.: Ibracimos. I. I.: Caple. R.: Froen. D.: Kreacer. A. Sinthesis 1989. 472 .

7. (a) Becker. D. P. Flynn. D. I. Tetrohedron Lett 1993, 34, 2087. (b) Becker, D. P.; Finn, D. T. Tetrohedron 1993, f9. 5047

8. (a) Reichwein. I. F.: Iacono. S. T.: Patel, M. C.: Pagcrikopt. B. I. Tetrahedron F.ett. 2002. 43. 3739. (b) Reichwein. J. F.: Iacono. S. T.: Pagenkopf. B. L. Te'tratedron 2002. 58. 3818.

9. Rao. M. L. N.: Periasany: M. .7. Organomet. (Them. 1997. 532. 143.

10. Kraftt, M. Г..; Bomaga, I.. V. R.; Wright. J. A.; Hirosawa, C. J. Org. Chem. 2002, 67, 12.3.3

11. Krafft, M. F.., Bomaga, I. V. R. Synlet 2000. 959.

12. Klemm. L. H.: Orchin. M. Int . . Chem Kinet 1997. 29. 473.

13. Son. S. U.: Lee. S. I.: Chung. Y. K.: Kim. S.W. Hyeon. T. Org. lett. 2002 . 277.

14. Krafti, M. Г..: Bomaga, I.. V. R.; Hirosawa, C. J. Org. Chem 2001. 66,3004 